\title{
Effect of Random Quenched Impurities on the Critical Behavior of a Four-State Potts System in Two Dimensions: An Experimental Study
}

\author{
L. Schwenger, K. Budde, C. Voges, and H. Pfnür \\ Institut für Festkörperphysik, Universität Hannover, \\ Appelstraße 2, D-30167 Hannover, Germany
}

(Received 17 March 1994)

\begin{abstract}
Critical behavior of the order-disorder phase transition of the $(2 \times 2)-2 \mathrm{H}$ structure on $\mathrm{Ni}(111)$ was investigated for the pure system and in the presence of $0.3 \%-3 \%$ of a monolayer of preadsorbed atomic oxygen. Depending on oxygen concentration, the values of the critical exponents $\beta, \gamma$, and $\nu$ change from those of the four-state Potts universality class to Ising-like exponents close to $T_{c}$, a behavior qualitatively in agreement with the effect of random quenched impurities.
\end{abstract}

PACS numbers: $68.35 . \mathrm{Rh}, 61.14 . \mathrm{Hg}, 64.60 . \mathrm{Fr}$

Phase transitions and critical phenomena in two dimensions (2D) have attracted wide interest in the past [1-3] because fluctuations play a much more important role than in $3 \mathrm{D}$ so that large deviations from mean field behavior are observed. Continuous order-disorder phase transitions of superstructures formed by adsorbed atoms or molecules give access to basically all universality classes that can be experimentally reached in $2 \mathrm{D}$ [4]. In particular, order-disorder phase transitions in the threeand four-state Potts universality classes can be and have been successfully studied $[5,6]$.

On the other hand, studies of phase transitions in adsorbed layers always face the problem of defects and impurities. Even without impurities the concentration of steps and point defects of the clean surface can hardly be reduced below the level of $0.1 \%$. In the simplest case, this leads to finite size effects; i.e., long range order and also correlations of critical fluctuations are disrupted by extended defects like steps. If coupling of fluctuations across steps occurs, the reduction of symmetry leads to corresponding changes of critical exponents, as observed for $\mathrm{O} / \mathrm{Ru}(0001)-p(2 \times 2)[7]$.

For the distribution of point defects the situation is less clear. For a system containing a random distribution of quenched impurities new stable critical points are predicted in the impure system with altered critical exponents in the case that $\alpha>0$, where $\alpha$ is the critical exponent of the specific heat [8], whereas the phase transition should remain unchanged for $\alpha \leq 0$. These predictions are corroborated by extended studies of the limiting case of the 2D Ising model $(\alpha=0)[9,10]$, and by Monte Carlo (MC) studies of the impure Baxter [11] and Baxter-Wu models [12]. Systematic experimental studies of the role of this type of defect for universality classes other than the Ising class, in particular of the three- and four-state Potts classes, are still missing.

The role of local defects is addressed in our study of the critical properties of the order-disorder phase transition of the $(2 \times 2)$ structure of atomic $\mathrm{H}$ on the $\mathrm{Ni}(111)$ surface, to which small concentrations of atomic oxygen were intentionally added. The phase diagram and the geometric structure of $\mathrm{H}$ in the ordered state are well known [13]. At surface temperatures $T_{s}$ above $100 \mathrm{~K}$ the $(2 \times 2)-2 \mathrm{H}$ structure (see inset of Fig. 1) is the only ordered phase with an ideal coverage of 0.5 monolayer (ML). Both threefold coordinated (fcc and hcp) sites are occupied [13]. According to the Landau rules [4], the order-disorder phase transition of this structure is expected to fall into the four-state Potts universality class, if the transition is continuous. Pure oxygen, on the other hand, is known to occupy only the fcc site at low coverage [14]. At the temperatures relevant for our investigation $(250-300 \mathrm{~K})$ and at the oxygen coverages used (0.003$0.03 \mathrm{ML}$ ) the pure oxygen system is above the ordering phase transition [15]. Assuming that the coadsorbed hy-

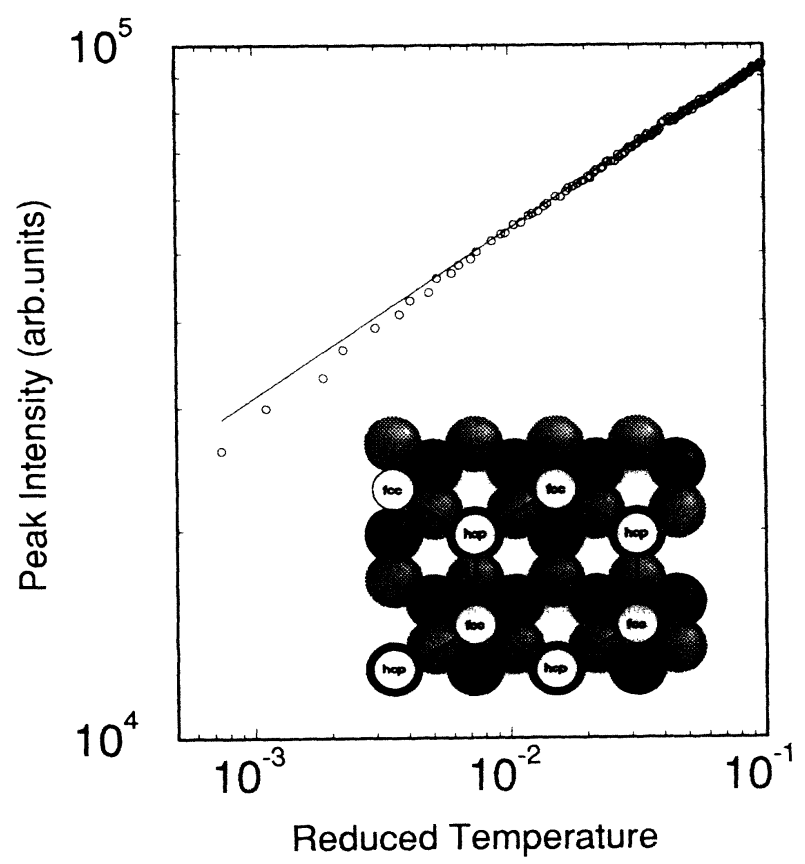

FIG. 1. Log-log plot of the peak intensity as a function of reduced temperature below $T_{c}$ for the pure hydrogen layer. The increase of slope close to $T_{c}$ is clearly visible. Inset: Schematic top view of the $(2 \times 2)-2 \mathrm{H}$ structure 
drogen phase does not strengthen the coupling between oxygen atoms, these therefore form a disordered lattice gas. Nevertheless, mobility of these $\mathrm{O}$ atoms seems to be low enough at the hydrogen critical point to represent essentially quenched impurities in the $\mathrm{H}$ layer, as shown below. No reaction between $\mathrm{H}$ and $\mathrm{O}$ atoms occurs up to the maximum temperature used in this study, as evident from the full reversibility of the phase transitions observed.

Experiments were carried out in a $\mu$-metal shielded UHV chamber (base pressure $3 \times 10^{-11} \mathrm{mbar}$ ). Low energy electron diffraction (LEED) profiles were measured using a high resolution instrument (SPA-LEED [16]) with an effective transfer width of $1200 \AA$. By use of liquid $\mathrm{N}_{2}$ and radiative heating or electron bombardment the sample temperature could be varied between 100 and $1400 \mathrm{~K}$. Only radiative heating was used for measurements. The sample temperature was measured and stabilized with a resolution of $0.01 \mathrm{~K}$ using a $\mathrm{NiCr}-\mathrm{Ni}$ thermocouple spot welded to the rim of the sample. Using a capillary array reproducibility of exposures of $0.5 \%$ was obtained for both $\mathrm{H}$ and $\mathrm{O}$, which was always preadsorbed at $T_{s}=$ $400 \mathrm{~K}$. The $\mathrm{Ni}$ sample $(20 \mathrm{~mm}$ in diameter) was cut by spark erosion from a Ni single crystal, precision oriented to about $0.02^{\circ}$, and polished with diamond pastes down to $0.25 \mu \mathrm{m}$ grain size. It was cleaned by extended cycles of $\mathrm{Ar}^{+}$sputtering and annealing. The clean surface -cleanliness was checked by Auger spectroscopycontains mainly monoatomic steps with an average step distance of $500-700 \AA$, as determined by LEED profile measurements.

Long range order in the $\mathrm{H}$ layers close to $\theta=0.5$ is limited essentially to one terrace, as concluded from the half-widths of the $(2 \times 2)$ superstructure spots far below $T_{c}$. Electron induced desorption or disorder was not detectable under our measuring conditions. Slow changes of $T_{c}$ caused by background adsorption of additional hydrogen could be reversed by annealing to $300 \mathrm{~K}$.

In order to avoid Fisher renormalization [17], we restricted ourselves to the maximum transition temperatures in all cases for a quantitative determination of the critical properties of the $(2 \times 2)$ order-disorder phase transition. $T_{c}$ was determined from the points of inflection of peak intensities versus temperature [18]. We obtained $T_{c}=267.2 \pm 0.3 \mathrm{~K}$ for the pure layer, which decreased to $263.5 \mathrm{~K}$ for the maximal coadsorbed oxygen coverage of $3 \%$.

First and second order superstructure spot profiles were measured in radial and in the corresponding perpendicular directions. For analysis they were parametrized using the following asymptotic form $[6,19]$ :

$S\left(\mathbf{q}_{\|}, t\right)=I_{0}(t) \delta\left(\mathbf{q}-\mathbf{q}_{0}\right)_{\|}+\frac{\chi_{0}(t)}{1+\left(\mathbf{q}-\mathbf{q}_{0}\right)_{\|}^{2} \xi^{2}(t)}+b g$.

These ideal profiles are convoluted with the instrument response function $\tau$, determined by low temperature superstructure profiles in the same directions as the measurements. $\mathbf{q}_{0}$ in Eq. (1) denotes a reciprocal lattice vector of the superstructure, and $t=\frac{T-T_{c}}{T_{c}}$ the reduced temperature. The first term describes the contribution from long range order, which close to $T_{c}$ behaves as $I_{0} \sim|t|^{2 \beta}$ below $T_{c}$. Above $T_{c}$, only the second term is left which is due to short range fluctuations with amplitude $\chi_{0} \sim|t|^{-\gamma}$ and correlation length $\xi \sim|t|^{-\nu}$. The last term denotes a constant background which was also fitted. Instead of numerical deconvolutions we carried out $2 \mathrm{D}$ fits to the experimental data [5].

Contributions of critical scattering to the profiles below $T_{c}$ were only observable at reduced temperatures $|t| \leq 10^{-3}$ so that no quantitative determination of the exponents $\gamma$ and $\nu$ was possible below $T_{c}$. Similiar observations have been made in $\mathrm{MC}$ simulations of $2 \mathrm{D}$ continuous phase transitions [20]. Therefore, peak intensities are not influenced by critical scattering for $|t|>10^{-3}$. Raw data of the peak intensity as a function of reduced temperature are plotted as log-log plot in Fig. 1 for the pure hydrogen layers. Simple power law behavior with an average slope of $0.11 \pm 0.01$ is only found in the range $5 \times 10^{-3}<|t|<0.1$, whereas the slope increases for smaller $|t|$. This effect could not be avoided by varying $T_{c}$ within the uncertainties of the determination of the point of inflection. Only the onset of the deviation in $t$ varies.

A similar effect is found for the data above $T_{c}$, where only critical scattering [second term of Eq. (1)] remains. No dependence of these data on the order of diffraction was seen, as expected for an order-disorder transition. The correlation lengths determined in different crystallographic directions also turned out to be isotropic within our experimental uncertainties. The amplitude of the deconvoluted peak profiles above $T_{c}$ and their inverse full widths at half maximum $\xi$ are again plotted as log-log plots in Fig. 2. Again an increase of slopes is seen at $t<5 \times 10^{-3}$, and a leveling off of both amplitudes $\chi_{0}$ and correlation lengths $\xi$ for $t<10^{-3}$. This latter effect can be well understood as finite size rounding induced by the steps on the surface. Indeed, our estimate of terrace lengths corresponds roughly to the constant value of $\xi$ at small $t$.

The data at larger $t$ can be well approximated by two sections of straight lines. The data for $t>5 \times 10^{-3}$ show power law behavior with effective exponents $\gamma=1.2 \pm 0.1$ for the amplitudes and $\nu=0.68 \pm 0.05$. These values agree very well with those expected for a continuous phase transition in the four-state Potts universality class. The increase of slope for $10^{-3}<|t|<5 \times 10^{-3}$ must be due to a mechanism different from finite size rounding, and might be caused by point defects already present on the clean surface.

In order to test this hypothesis we introduced a controlled amount of preadsorbed oxygen into the system. 


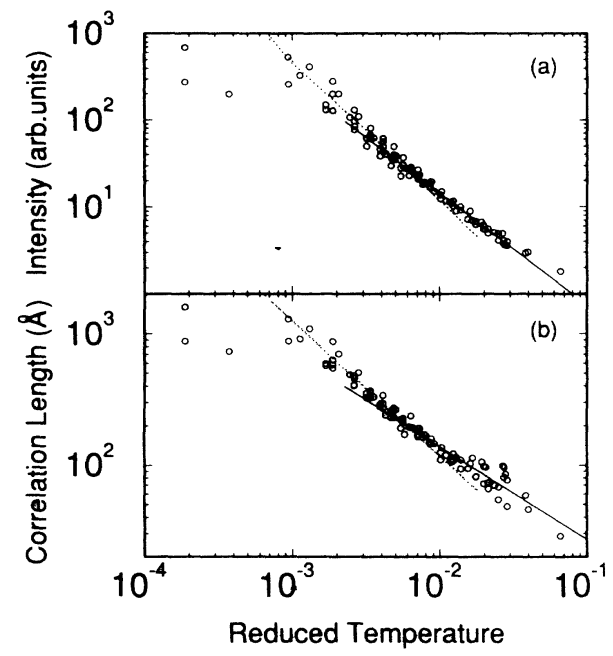

FIG. 2. Representative log-log plots of the amplitudes of critical scattering (a) and of the correlation lengths of first and second order superstructure beam profiles above $T_{c}$. Slopes of straight lines correspond to effective exponents $\gamma=1.61$ (dotted line) and $\gamma=1.28$ (solid line) for the upper and lower section of (a), respectively. For $\nu$ the values 1.02 and 0.71 are obtained from (b).

$0.3 \%$ of a monolayer of oxygen did not essentially alter the behavior of the "clean" surface, whereas we observed a significant shift of the crossover between the two straight sections in Fig. 2 to approximately $t=1.5 \times 10^{-2}$ already at a concentration of $0.007 \mathrm{ML}$. At the highest concentration of oxygen tested $(0.03 \mathrm{ML})$ no crossover is visible any more. This situation is shown in Fig. 3. Again taking the point of inflection of peak intensity versus temperature curves as $T_{c}$, we obtain effective exponents $\gamma=1.68 \pm 0.15$ and $\nu=1.03 \pm 0.08$. Similarly, the effective exponent $\beta$ increases slightly from the value 0.11 without oxygen to $0.135 \pm 0.01$ at $3 \%$ oxygen coverage. Errors are mainly caused by the uncertainty in $T_{c}$. Our results are summarized in Table I.

They show that our data can be analyzed consistently, i.e., for the pure system and with all concentrations of oxygen impurities tested, by two regimes in $t$, which both can be described by effective critical exponents. A sys-

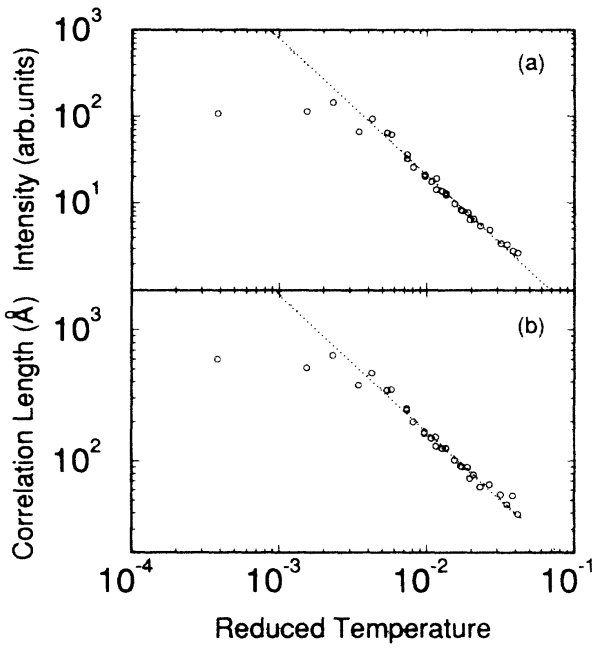

FIG. 3. Same as Fig. 2, but with $3 \%$ of a monolayer of preadsorbed atomic oxygen. Effective exponents as determined from the straight lines drawn are $\gamma=1.63$ and $\nu$ $=1.01$.

tematic shift to higher values of $t$ of the crossover between the two regimes is found as a function of oxygen concentration.

At large $t$ critical exponents of a pure four-state Potts system are obtained within error bars (see Table I). There is a slight tendency in these experimental values to exceed the theoretical ones, especially for the exponent $\beta$ of the order parameter. Obviously, logarithmic corrections to scaling, expected for this universality class [21], are relevant in this case, in contrast to systems in the same universality class studied earlier $[5,6]$. This is not totally unexpected because of the different local structure in the $(2 \times 2)-2 \mathrm{H}$ unit cell compared to the systems with $p(2 \times 2)$ unit cells investigated earlier.

Obviously, already the pure system contains local defects, which affect the critical exponents close to $T_{c}$ in very much the same way as the added oxygen atoms. Because of the oxygen induced increase in the concentration of defects, the crossover between the two regimes is shifted to smaller $\xi$, i.e., to larger $t$ values. At the

TABLE I. Averages of experimentally determined effective critical exponents and comparison with theoretical expectations for the four-state Potts and the Ising universality classes (see Ref. [23]). "Pure" means exponents determined from those sections of the experimental data not influenced by the oxygen impurities. Exponents from the impurity dominated parts are labeled "impure."

\begin{tabular}{lcccc}
\hline \hline & \multicolumn{2}{c}{ Experiment } & \multicolumn{2}{c}{ Theory } \\
& Pure & Impure & Ising & Four-state Potts \\
\hline$\beta$ & $0.11 \pm 0.01$ & $0.135 \pm 0.01$ & 0.125 & 0.083 \\
$\gamma$ & $1.2 \pm 0.1$ & $1.68 \pm 0.15$ & 1.75 & 1.167 \\
$\nu$ & $0.68 \pm 0.05$ & $1.03 \pm 0.08$ & 1.0 & 0.667 \\
\hline \hline
\end{tabular}


smallest concentration of oxygen tested it seems that the oxygen atoms are preferentially adsorbed at the local defects already present on the surface. Thus no additional defects are created, and the phase transition remains unchanged.

The changes of critical exponents are consistent with the effect of random quenched impurities. Also the impurity induced slight decrease of $T_{c}$ is compatible with this model [22]. According to the Harris criterion [8] the exponents should be changed for a system within the four-state Potts universality class, where $\alpha$ is positive ( $\alpha=2 / 3$ [23]). Observations very similar to our experimental findings have been made for random quenched bond and/or site disorder in $\mathrm{MC}$ simulations of the Baxter-Wu model [12], which also belongs to the fourstate Potts class, and of the Baxter model with various positive values of $\alpha$ [11]. In the impure systems critical exponents close to those of the Ising class were obtained in all cases, in good agreement with our experimental findings. It seems therefore that the effect of random quenched impurities to lead to Ising-like critical exponents is not just limited to a few special cases. However, to our knowledge no theoretical justification for these findings can be given at the moment, nor is it clear that the critical exponents found really belong to the Ising universality class. Annealed impurities, on the other hand, are not compatible with our experimental data. Fisher renormalization should then be found even at symmetry points of the hydrogen phase diagram $[17,22]$ with resulting effective exponents close to $T_{c}$ much larger than those found experimentally.

Summarizing, we have found that the order-disorder phase transition of the $(2 \times 2)-2 \mathrm{H}$ structure on $\mathrm{Ni}(111)$ is continuous, and yields critical exponents for the pure layers which are compatible with those of the four-state Potts universality class. These critical exponents are modified in the same way by point defects already present on the bare surface and by small amounts of preadsorbed oxygen. These findings are compatible with the model that both the defects on the bare surface and single oxygen atoms or small clusters of them act as random quenched impurities within the hydrogen layer. These effects can be separated from those of extended defects like steps, which lead to finite size rounding.

This work was supported by the Deutsche Forschungsgemeinschaft through project Pf238/1.

[1] E. Bauer, in Topics in Current Physics, edited by W.
Schommers and P. von Blanckenhagen (Springer, Berlin, 1987), Vol. 43, p. 115.

[2] K. Binder and D. P. Landau, in Molecule-Surface Interaction, edited by K. Lawley (Wiley, New York, 1989), p. 91.

[3] B. N. J. Persson, Surf. Sci. Rep. 15, 1 (1992).

[4] M. Schick, Prog. Surf. Sci. 11, 245 (1981); C. Rottman, Phys. Rev. B 24, 1482 (1981).

[5] P. Piercy and H. Pfnür, Phys. Rev. Lett. 59, 1124 (1987); H. Pfnür and P. Piercy, Phys. Rev. B 40, 2515 (1989).

[6] M. Sokolowski and H. Pfnür, Phys. Rev. B 49, 7716 (1994).

[7] M. Sokolowski and H. Pfnür, Phys. Rev. Lett. 63, 183 (1989); M. Sokolowski, H. Pfnür, and M. Lindroos, Surf. Sci. 278, 87 (1992).

[8] A. B. Harris, J. Phys. C 7, 1671 (1974).

[9] B. N. Shalaev, Phys. Rep. 23, 131 (1994)

[10] J. S. Wang, W. Selke, V. L. Dotsenko, and V. B. Andreichenko, Physica (Amsterdam) 164, 221 (1990).

[11] D. Matthews-Morgan and D. P. Landau, Phys. Rev. Lett. 53, 679 (1984).

[12] M. A. Novotny and D. P. Landau, Phys. Rev. B 24, 1568 (1981).

[13] K. Christmann, R. J. Behm, G. Ertl, M. A. Van Hove, and W. H. Weinberg, J. Chem. Phys. 70, 4168 (1979); L. Hammer, H. Landskron, W. Nichtl-Pecher, A. Fricke, K. Heinz, and K. Müller, Phys. Rev. B 47, 15969 (1993).

[14] E. Schmidtke, C. Schwennicke, and H. Pfnür, Surf. Sci. (to be published); D. T. Vu Grimsby, Y. K. Wu, and K. A. R. Mitchell, Surf. Sci. 232, 51 (1990); M. A. Mendez, W. Oed, A. Fricke, L. Hammer, K. Heinz, and K. Müller, Surf. Sci. 253, 99 (1991).

[15] A. R. Kortan and R. L. Park, Phys. Rev. B 23, 6340 (1981).

[16] U. Scheithauer, G. Meyer, and M. Henzler, Surf. Sci. 168, 441 (1986).

[17] M. E. Fisher, Phys. Rev. 176, 257 (1968).

[18] N. C. Bartelt, T. L. Einstein, and L. D. Roelofs, Phys. Rev. B 32, 2993 (1985).

[19] H. E. Stanley, Introduction to Phase Transitions and Critical Phenomena (Oxford University Press, New York, 1971).

[20] V. Privman, P. C. Hohenberg, and A. Aharony, in Phase Transitions and Critical Phenomena, edited by C. Domb and J. L. Lebowitz (Academic Press, London, 1991), Vol. 14, p. 1; N. C. Bartelt, T. L. Einstein, and L. D. Roelofs, Phys. Rev. B 35, 1776 (1987).

[21] J. L. Cardy, M. Nauenberg, and D. J. Scalapino, Phys. Rev. B 22, 2560 (1980).

[22] R. B. Stinchcombe, in Phase Transitions and Critical Phenomena, edited by C. Domb and J. L. Lebowitz (Academic Press, London, 1983), Vol. 7, p. 151.

[23] F. Y. Wu, Rev. Mod. Phys. 54, 235 (1982). 


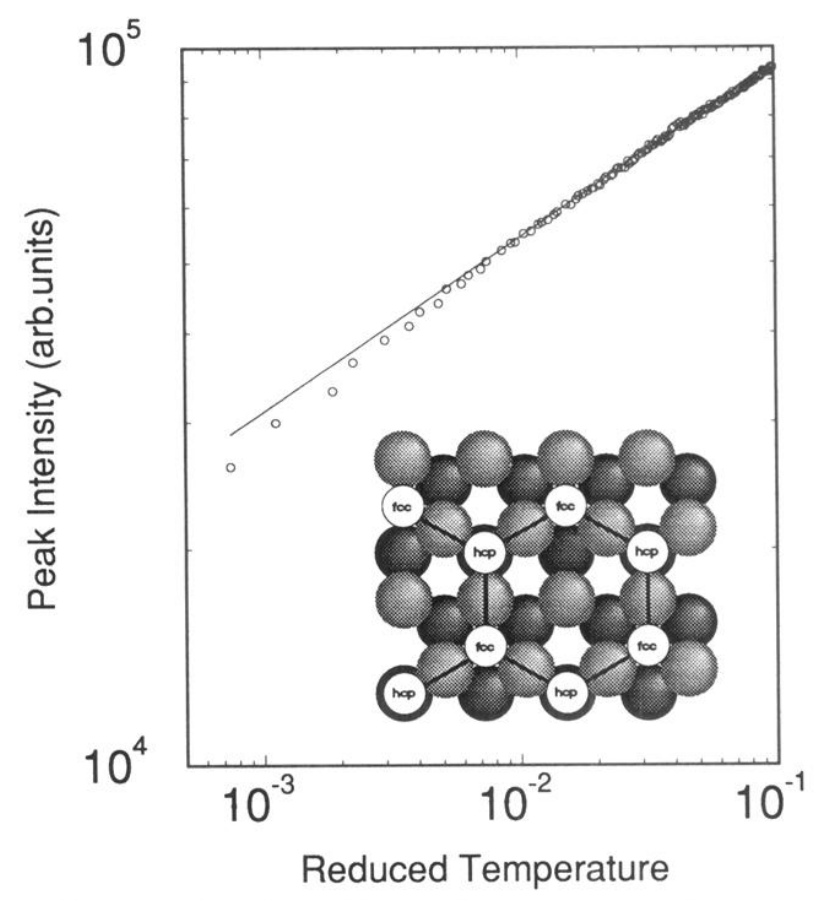

FIG. 1. Log-log plot of the peak intensity as a function of reduced temperature below $T_{c}$ for the pure hydrogen layer. The increase of slope close to $T_{c}$ is clearly visible. Inset: Schematic top view of the $(2 \times 2)-2 \mathrm{H}$ structure. 